Mediterranean Journal of Humanities mjh.akdeniz.edu.tr I/ 1, 2011, 53-61

\title{
Die Keramikfunde des Görükle-Grabes in Bursa
}

\author{
Görükle Tümülüs Mezarından Ele Geçen Seramikler
}

\begin{abstract}
Mustafa BULBA*
Zusammenfassung: Das Thema dieses Aufsatzes befasst sich mit Funde eines Grabmals auf dem Campus der Uludağ-Universität, welches während der Bauarbeiten zufällig entdeckt wurde. Die vollständige Publikation dieses Grabes liegt in Kurze vor. Bei dieser Arbeit werden nur die Keramikfunde diskutiert. In diesem Grab sind sowohl Feinkeramik als auch Grobkeramik gefunden. Besonders die fehlende Publikation über die Grobkeramik der hellenistischen Periode erschwert eine genaue Ordnung dieser Gefäße innerhalb der hellenistischen Periode. Das Grab wird vor allem durch eine Münze aus der Regierungszeit Prusias II in die erste Hälfte des 2. Jhs. datiert.
\end{abstract}

Schlüsselwörter: Görükle, tumulus, Hellenistische Keramik, Lagynos, napf, lampe, oinochoe, amphora

Özet: Bursa'nın Nilüfer İlçesi'ne bağlı Görükle Köyü'ne yapılan yeni hal inşaatının çevre düzenlemesi sırasında bir tümülüs mezar açığa çıkarılmıştır. Yapılan kurtarma kazılarında ele geçen seramikler ve bir adet sikke Bursa Müzesi'ne getirilmiştir. Yapılan kurtarma kazılarında mezarın içerisinde tek bir gömü tespit edilmiştir. Ele geçen sikke sayesinde mezar İÖ. II. yüzyılın ilk yarısına, II. Prusias dönemine tarihlendirilmektedir. Mezarda ele geçen seramik eserler hamur, biçimlendirme ve bezeme bakımından yerel özellikler göstermektedirler ve kaliteleri oldukça düşüktür. Bununla birlikte dönemin genel özelliklerini yansıtmaktadırlar. Tarihlendirmeleri ele geçen sikke sayesinde güvenilir bir şekilde yapılabilmektedir. Bu eserler bölgede ele geçen ve henüz yayınlanmayan birçok eserin de tarihlendirilebilmelerine $1 s ̧ 1 k$ tutacaktır.

Anahtar Kelimeler: Görükle, tümülüs, Hellenistik Dönem Seramiği, Lagynos, kase, kandil, oinochoe, amphora

\section{Keramikfunde}

1- Lagynos hat einen breiten $\mathrm{Fu}$ (Abb. 1, Zei. 1: H: 26,6 cm. T: bräunlicher Ton mit wenig Sand und Kalk. Die Verzierungen sind mit rot auf weißem Überzug übertragen. B: Ganz erhalten. Die Außenseite ist weitgehend mit Kalk überzogen. Nur auf einem kleinen Teil sieht man die Gefäßoberfläche bzw. die Verzierungen. Die Mündung ist außen etwa $2 \mathrm{~cm}$ bemalt. Die Stelle zwischen dem Hals und der Schulter mit einem dünnen Band verziert. Gleich darunter, auf der Schulter, an beiden Seiten des Henkels, befinden sich zwei ungleichmäßige vertikale Bänder (Auf der linken Seite sieht man nur ein Band). Gleich unter dem unteren Henkelansatz befinden sich 3 horizontale Bänder, von denen der mittlere dicker ist. Der weiße Überzug ist bis auf wenigen Teilen abgelaufen). Der breite Körper macht auf der Schulter und unter dem Bauch mit weichen Biegungen Krümmungen. Der Körper verbreitet sich leicht nach oben hin und verbindet sich mit einer leichten Krümmung mit dem Hals. Der hohe Hals verjüngt sich leicht nach oben. Die Mündung biegt sich mit einer scharfen Kante nach Außen. Der Rand der Lippe

\footnotetext{
* Yrd. Doç. Dr., Akdeniz Üniversitesi, Edebiyat Fakültesi, Arkeoloji Bölümü, Antalya, mbulba@akdeniz.edu.tr
} 
ist scharfkantig und senkrecht geformt. Der bandförmige Henkel zeigt auf der Außenseite, in der Mitte, eine Wölbung im Schnitt. Er sitzt an der breitesten Stelle des Körpers und erhöht sich leicht nach außen gebogen aufrecht und verbindet sich mit oben nach leichter Biegung heruntergehend mit dem oberen Teil des Halses.

Der Körper ist, soweit man erkennt, gänzlich mit beiger Farbe überzogen. Die Ornamente sind mit roter Farbe gegeben. Im kalkfreien Bereich sieht man an beiden Seiten des Henkels, von der Linie zwischen Hals und dem Körper herunterfallend je zwei wellige haarschleifenförmige Bänder. Der Bauch unter dem Henkelansatzes ist mit dicken und dünnen horizontalen Bändern versehen. Die Mündung ist im Innen und Außen bemalt. Die restlichen Verzierungen sind wegen dem Kalk auf der Oberfläche des Gefäßes nicht zu erkennen.

Der Hauptfundort dieser Form erstreckt sich von Westkleinasien bis zum nördlichen Schwarzmeergebiet, von Griechenland über ägäischen Inseln bis nach Ägypten (VessbergWestholm, 1956, 59; Cook, 1966, 207 ff; Schäfer, 1968, 101; Bruneau, 1970, 251 f; 1980, 10 f; Mitsopoulos-Leon, 1991, 75 f; Gürler, 2002, 542 f). Selbst in Italien wurden relativ viele weißgrundige Lekythoi gefunden (Schmidt, 1985, 66). Obwohl die Form in so einer breiten gefunden wird, konnte man bisher über ihre Herstellungszentrum (Herstellungszentren?) keine Anhaltspunkte feststellen. Allgemein werden angenommen, dass sie im Ostgriechenland, auf ägäischen Inseln, Westkleinasien oder auf Zypern hergestellt wurden (Schäfer, 1968, 109; Schmidt, 1985, 66).

Schmidt hat eine Dissertation über die einzelne Formen hellenistischer Keramik geschrieben und hat dabei auch die Entwicklungsgeschichte des Lagynos sorgfältig diskutiert. Ich will hier den Teil über Lagynoi von seiner Arbeit zusammenfassen und den Lagynos im Görükle-Grab auf diese Weise datieren: Die Verzierungen der Lagynoi befinden sich meistens auf der Schulter und am Hals und sie werden sich von horizontalen Bändern begrenzen. Als Ornamente werden sich Efeumotiven, Daphneblätter und Zungenmotiven bevorzugt. Sehr selten sieht man auch Figuren wie girlandtragende Eroten auf Lagynoi.

Unverzierte Lagynoi gehören meistens in die 2. Hälfte des 3. Jhs. und in die erste Hälfte des 2. Jhs. Bei diesem Typ sieht man eine markante Linie auf der Schulter. Diese Linie ist zwar gut betont aber auch nicht ganz scharfkantig, sondern leicht gerundet. Der Bauch erhöht sich vom engen Fuß stark nach oben hin bis zum Schulter und der Körper bekommt somit beinahe eine dreieckige Form. Die breite Schulter verbindet sich schwer mit dem Bauch und zeigt eine Gegenstärke zum Bauch und aber eine gute Harmonie mit dem Hals, der sich nach oben hin verjüngt. In der hochhellenistischen Periode wird der gedrungene Körper leicht erhöht und sich mit einer weichen Rundung mit der Schulter verbindet. Zwischen den Bindungslinien zwischen dem Bauch-Schulter und Schulter-Hals entsteht eine gestreckte ,S-Linie'. Auch der Hals zeigt in diesem Zeitraum eigene Besonderheiten. Der Hals verbindet sich mit der Schulter nicht röhr artig, sondern ist jetzt sein unterer Teil breiter und zeigt zur Biegung des Halses eine Harmonie. Außerdem zeigen Henkel nicht mehr winkelige Formen, sondern zeigt durch ihre gerundeten Biegungen Ausgleich mit den gesamten Körperlinien. In der früheren sind Ornamente auf den Hals beschränkt, während in der hochhellenistischen Periode auch der Bauch verziert wurde. In der spätesten Phase werden die Hälse sich nicht mehr nach oben hin verjüngt, sondern sind sie zylindrisch. Die weißgrundigen Lagynoi werden ab der Mitte des 2. Jhs hergestellt. Nach Funde aus Delos waren sie noch im 2. Viertel des 1. Jhs. v. Chr. immer noch in Gebraucht. Aus der römischen Periode sind bisher keine Beispiele bekannt.

Nach heutigen Forschungsstand kann man also annehmen, dass sie ab der Mitte des 1. Jhs. v. Chr. nicht mehr hergestellt wurden. Lagynoi sind weitgehend in den heiligen Festen gebraucht worden, und haben zum Weintrinken gedient. Auch die Ornamente zeigen, dass sie vor allem bei den dionysischen Festen in Gebrauch waren. Sie wurden auch öfters als Grabbeigaben gedient, sind aber nebenbei auch bei täglichem Leben in Gebrauch, wie wier aus den 
Fundumständen herausfinden können (Schmidt, 1985, 66 f).

Aus diesen Besonderheiten können wir feststellen, dass unser Gefäß mit seinem hohen Körper mit runden Biegungen und sich nach oben verjüngendem Hals zur hochhellenistischen Periode gehört. Wenn wir davon ausgehen, dass die weißgrundigen Lekythoi ab der Mitte des 2. Jhs. v. Chr. in Gebrauch waren, dürfen wir so annehmen, dass unser Lagynos so etwa in der Mitte des 2. Jh. v. Chr. hergestellt wurden könnte.

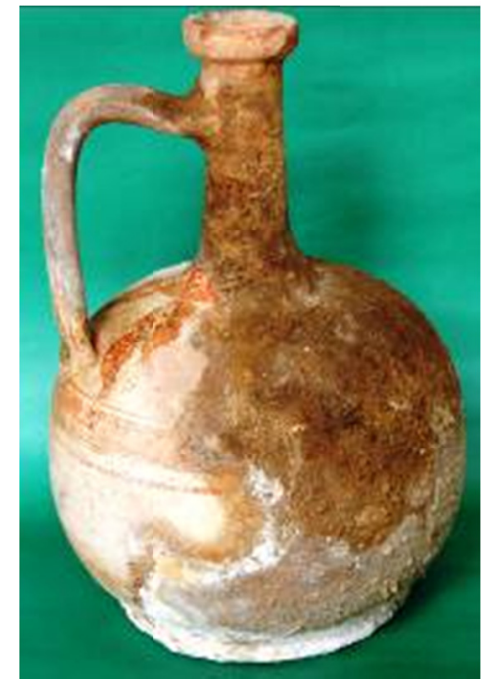

Abb. 1

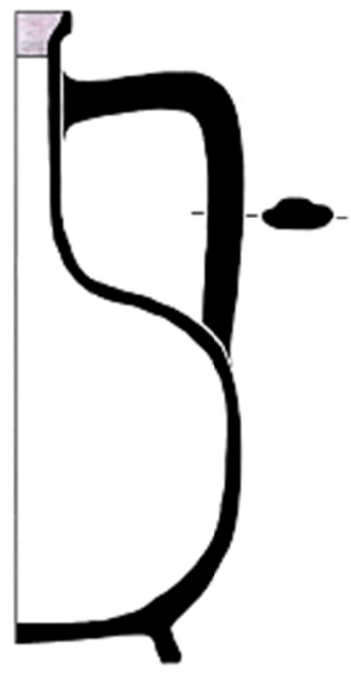

Zei. 1

2- Etwa halbkugelförmig $\boldsymbol{N a p f}$ hat ohne Fuß, die Standfläche ist glatt (Abb. 2, Zei. 2: H: 6,5 $\mathrm{cm}$. T: sehr hart, dunkelrotfarbiger Ton. Sehr wenig Glimmer. Kamelhaarfarbiger Überzug. B Ganz erhalten. Innen- und Außenseite mit Kalk überzogen. Wegen der zu harten Brennung sieht man Verzögerungen auf dem Gefäß). Der Körper erhöht sich mit breiter Biegung nach oben bis zu einer dünnen, plastischen Linie. Über dieser Linie verbindet sich der Körper mit der Mündung nach einer konkaven Biegung. Da dieser Napf eine dünne Linie am Körper hat und mit der Kupferfarbe bemalt ist, liegt nahe, dass er nach Metallvorbildern angefertigt wurde. Qualitativ zeigt er, abgesehen vom Lagynos, ein höheres Niveau unter den Keramikfunden in diesem Grab.

Seine besten Vergleichsbeispiele, unter den publizierten Verwandten aus den näheren Bereichen, kommen aus Sardis. Weil sie in der Form mit den akamenidischen Näpfen sehr ähnlich sind, werden auch diese Gefäße, Akamenidische Näpfe' genannt und kommen überall vor, wo die Akameniden ansässig waren oder Einfluss hatten.

Die besten Vergleichsbeispiele für den Napf aus dem Görükle-Grab kommen aus Daskyleion (Dusinberre, 1999, 73 f; Rotroff-Oliver, 2003, 60 f). Auch die Näpfe aus Sardis zeigen nahe Verwandtschaften mit diesem Gefäß. Nach Schätzungen der Gräber von Sardis wurden sie in dieser Stadt von zwei unterschiedlichen Werkstätten hergestellt. Die Bezeichnungen dieser Näpfe zeigen Verwandtschaften mit unserem Gefäß. Rotroff und Oliver bezeichnen sie, von guter Qualität, Glimmer enthaltend, sowohl der Ton als auch der Überzug rotfarbig und der Überzug schwach haltend' (Dusinberre, 1999, 77, Fig. 2). Alle diese Besonderheiten kommen auch bei unserem Napf vor. Obwohl die Formen ziemlich ähnlich sind, zeigt unser Napf keine so starke konkave Biegung unter der Lippe wie bei den Näpfen aus Sardis, sondern sie ist mehr aufrecht und nur leicht konkav. Allerdings sieht man auch unter den publizierten Näpfen aus Sardis unterschiedliche Variationen, so dass auch unser Napf in Sardis oder in einer Werkstatt unter dem Einfluss von Sardis hergestellt worden sein könnte. 
Die Näpfe aus Sardis werden mit großer Wahrscheinlichkeit vor 213 v. Chr. Datiert (Rotroff-Oliver, 2003, 61). Da unsere Münze in der Regierungszeit von Prusias II geprägt wurde (182-149), kommt es etwa zu einem Zeitunterschied, zwischen den jüngsten $\mathbb{N a ̈ p f e n ~ a u s ~ S a r d i s ~}$ und unserem Napf, von mindestens 30 Jahren, der aber akzeptabel ist. Es ist bekannt, dass manche Gefäße nach langer Zeit als Grabbeigaben verschenkt wurden. Es ist außerdem ebenfalls möglich, dass unser Napf in einer jüngeren Periode von einer anderen Werkstatt hergestellt wurde. Denn es ist eher wahrscheinlich, dass dieser Napf wie die anderen Gefäße aus diesem Grab, in der ersten Hälfte des 2. Jhs. hergestellt wurde. Die jüngsten Beispiele dieser Art werden jedoch in Sardis zum Ende des 3. Jhs. v. Chr. datiert (Fundgruppe 7 und 8; RotroffOliver, 2003, 61).

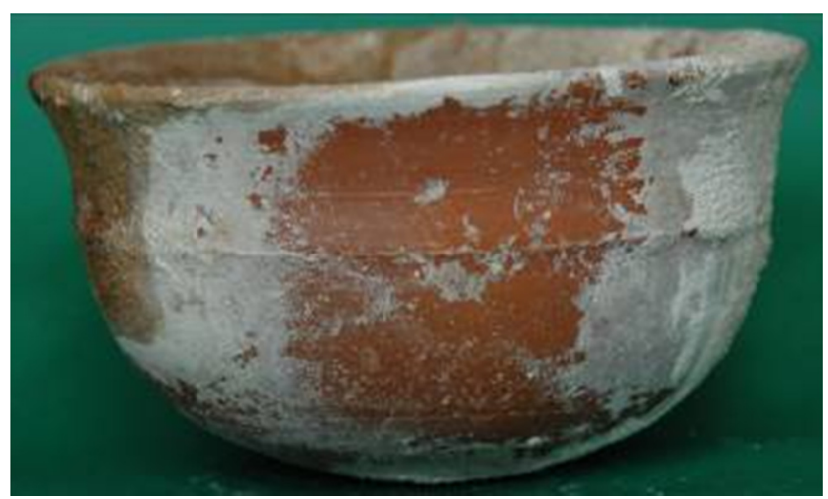

Abb. 2

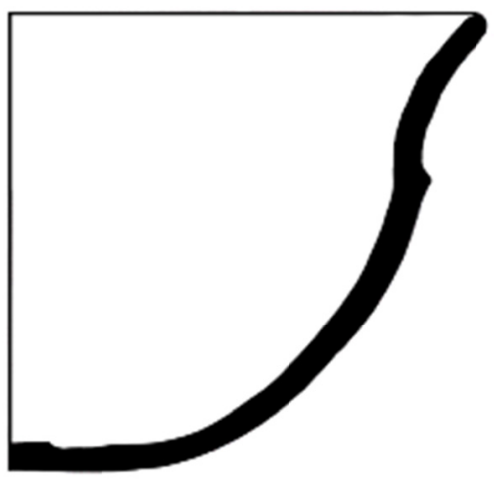

Zei. 2

3- Diese Trinkschale verbreitert sich unter der Lippe mit einer geraden Linie erstmal nach Außen und verjüngt sich wieder zum Fuß hin (H: 9,1 cm. T: hart gebrannt. Dunkelziegelfarbiger Ton mit Sand und Kalkpartikeln. Ohne Bemalung und Überzug. B: abgesehen von der Hälfte eines trommelförmigen Henkels ganz erhalten An der Außenseite sieht man große Kalkplatten auf der Oberfläche, die Innenseite zeigt weitgehend Rußspuren und ist an wenigen Teilen mit Kalkplatten bedeckt. Wegen der harten Brennung zeigt das Gefäß an manchen Teilen, besonders an der Mündung, Deformationen. Die Henkel haben plastische Beifügungen, die trommelförmig sind.) und ähnelt somit den knidischen Schalen (Abb. 3, Zei. 3: Dusinberre, 1991, 91; Elaigne, 2002, 172, Abb. 10; Kögler, 2010, 103, Abb. A -Form E. Mit Ringhenkel-). Er ist allerdings höher und sein ringförmiger Fuß hat ein größeres Volumen als die knidischen Schalen. Außerdem hat die Verbreiterung unter der Lippe eine leicht nach innen gewölbte Biegung. Den großen Unterschied im Vergleich zu den knidischen Schalen mit Pi-Henkeln bilden die vertikalen Henkel. Diese genannten Besonderheiten geben diesem Gefäß eine Mischform zwischen Kylix und Kantharos. Nebenbei haben die knidischen Schalen an der Innenseite und Außen unter der Mündung einen Überzug, während unsere Schale nirgendwo auf dem Körper Überzugsspuren zeigt. Mit ihren scharfen Zügen und plastischen TrommelAnsätzen soll dieses Gefäß wie bei den knidischen Schalen Kopien der Metallvorbilder sein. E. Doksanaltı hat die Schalen aus Knidos publiziert und festgestellt, dass sie besonders im 2. Jhd. v. Chr. sehr populär waren. Doksanaltı teilt außerdem mit, dass sie mit der Zeit nur sehr geringe Formentwicklungen zeigen und ihre scharfen Konturen in der römischen Periode verlorengehen und sich zu einem konvexen Vorsprung entwickelten.

Ein ähnliches Gefäß wie das unsrige, jedoch mit vertikalen Henkeln, wurde auch in Athen gefunden, auch wenn sie nicht ganz nah verwandt sind, wo die knidischen Schalen auch kopiert wurden (Doksanalt1, 2003, 27 f; Rotroff, 1997, 119). Die Kopien der knidischen Schalen tauchen in Athen ab Anfang des 2. Jhds. v. Chr. auf und zeigen ebenfalls mit der Zeit kleine Formänderungen wie bei den knidischen Schalen (Doksanalt1, 2003, 28). Das Vergleichsbei- 
spiel für unsere Trinkschale ist eine Ausnahme unter den Funden in Athen und wurde von den Verfassern wegen ihre Qualität und der Ornamente in eine etwas frühere Periode, zum Ende des 3. Jhds. v. Chr. datiert. Unser Gefäß soll aber wegen seiner bescheidenen Qualität und dem fehlenden Überzug eine lokale Kopie der knidischen Schalen sein, weil in Knidos selbst und in Athen, wo die knidischen Schalen kopiert wurde, diese Gefäße nur kleine Formänderungen zeigen, können wir auch unser Gefäß nicht in einen bestimmten Zeitraum setzen. Da die Münze aus diesem Grab spätestens 149 v. Chr. hergestellt wurde, dürfte auch dieses Gefäß in der ersten Hälfte des 2. Jhds. produziert worden sein.

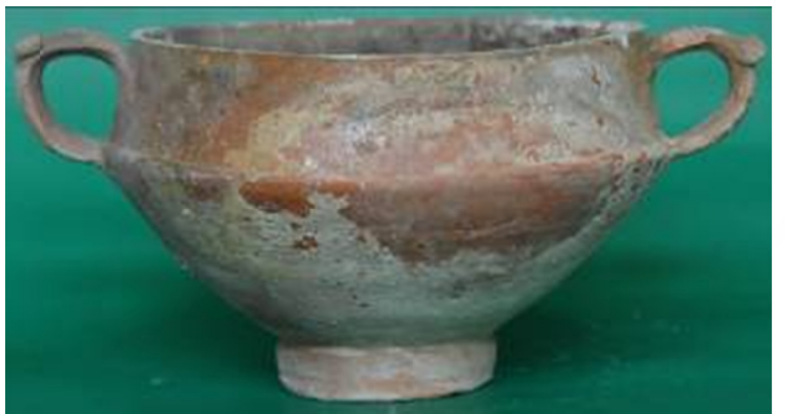

Abb. 3

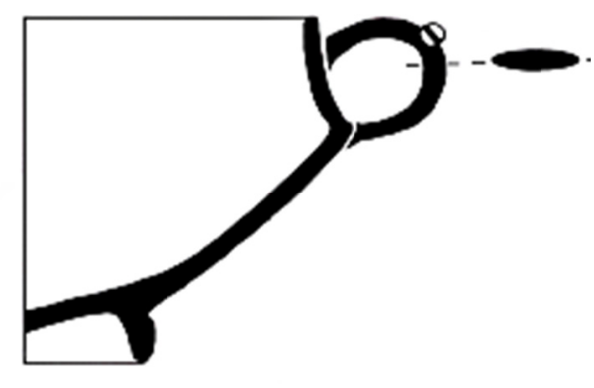

Zei. 3

4- Die Form dieser kleinen Lampe wurde gepresst und hat qualitativ relativ guten Ton und ist von einer guten Arbeitsqualität (Abb. 4, Zei. 4: H: 2,6 cm. T: Bräunlicher Ton mit Sand. Malfarbe schwarz. Durch die Eintauchung gemalt. B Abgesehen vom Henkel ganz erhalten. Auf manchen Flächen sieht man neue Zerstörungsspuren. Die Löcher im Diskus und an der vorderen Seite zeigen Verzerrungen. An der breitesten Stelle des Diskus und in der Mitte zwischen diesem Bereich und der Mündung befinden sich an den beiden Seiten je zwei plastische Vorsprünge. Unter dem Fuß sieht man unregelmäßige Farbspuren, die durch die Eintauchung entstanden sein könnte). Im hinteren Teil befand sich der vertikale, bandförmige Henkel, der jetzt fehlt. Besonders geben die herzförmigen plastischen Verlängerungen in Form der stilisierten Efeu-Motive an der breitesten Stelle des Diskus, sowie zwischen der breitesten Stell und dem Loch der Lampe, dieser eine elegante Erscheinung.

Unter den publizierten Lampen sind mir keine guten Vergleichsbeispiele bekannt. Herzblattlampen sind zahlreich in Pergamon gefunden worden. Sie haben gleichfalls an den breitesten Stellen Herzblattornamente, die an Efeu erinnern (Schäfer, 1968, 130 f). Allerdings sie haben nicht wie bei unserer Lampe eine flache Oberfläche, sondern ein doppelkonvexes Profil. Außerdem sind ihre Oberflächen reichlich geschmückt, obgleich unser Beispiel nur schlicht mit Efeu und Herzornamenten verziert ist. Auch die Efeumotive zwischen den Herzornamenten und den Schnauze sieht man bei den Lampen aus Pergamon nicht. Solche Ornamente sieht man allerdings nur für die pergamenische oder westkleinasiatische Form bezeichnend (Schäfer, 1968, 130, Fußnote 3). Eine Lampe mit einem Herzornament auf einer Seite wurde in Notion gefunden und wird jetzt im Ephesos-Museum aufbewahrt (Rotroff, 1997, 119, Fig. 23-405). Ein ähnliches Gefäß zur Lampe aus Notion wurde in Tsamourli bei Samsun, jetzt im British Museum ausgestellt, gefunden, welche vom Verfasser ins 2. Jh. v. Chr. datiert wurde und als Herkunftsort Kleinasien angenommen (Rotroff, 1997, 119) wird. Es wird außerdem von Tuluk berichtet, dass eine andere vergleichbare Lampe mit der Lampe aus Notion verwandt wäre, die jetzt auch im Ephesos-Museum aufbewahrt (Tuluk, 2003, 23) wird. Er berichtet, dass er keine anderen Vergleichsbeispiele für diese Lampen mit Herzornament kennt. Ich will hier einige weitere Vergleichsbeispiele aus dem British-Museum vorschlagen, die von Bailey publiziert wurden. Als Herkunft wurde Ägypten angegeben und sie haben gleichifalls herzförmige Vorsprünge (Bailey, 1975, 79-80, Pl. 26). Besonders die Lampe Q590 EA ähnelt weitgehend 
unserer Lampe, hat allerdings keine Vorsprünge zwischen der Schnauze und dem Diskus wie bei unserem Beispiel. Diese Lampe aus dem British-Museum wird zum Ende des 2. Jhds. oder ins 1. Jhd. v. Chr. datiert. Ich will unsere Lampe wegen der Münze aus diesem Grab zur ersten Hälfte des 2. Jhds. datieren. Ihr Herstellungszentrum kann man allerdings wegen zu weniger Hinweise nicht genau feststellen. Die Lampen, sowohl aus Ägypten als auch aus Notion, zeigen keine genauen Ähnlichkeiten mit unserem Beispiel, bilden aber trotzdem, soweit mir bekannt ist, beste Vergleichsbeispiele. Es wäre aber theoretisch möglich, dass diese Lampe in der chaotischen hellenistischer Periode durch militärische Züge aus Notion oder sogar aus Ägypten gekommen ist. Mit größerer Wahrscheinlichkeit wurde sie aber als Imitation der pergamenischen Lampen, die in großer Anzahl mit verschiedenen Variationen in allen Phasen der hellenistischen Zeit produziert wurden (Schäfer, 1968, 131 f), in einer Werkstatt in der Umgebung des Grabes hergestellt.

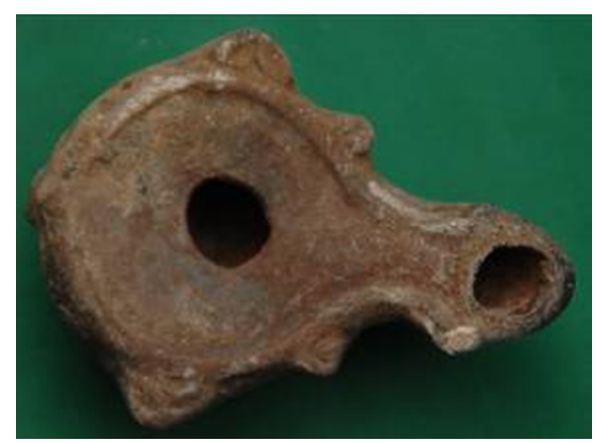

Abb. 4
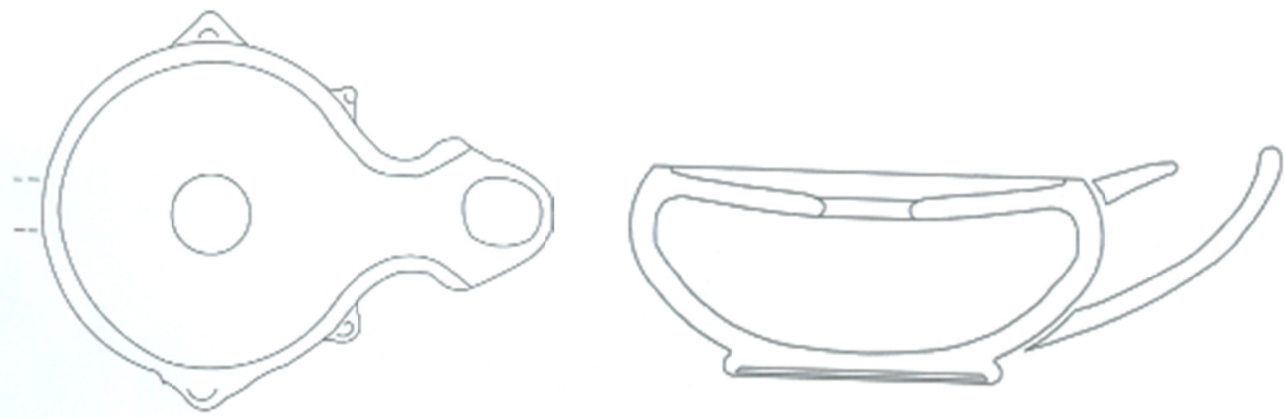

Zei. 4

5- Oinochoe mit ihrem breiten, leicht langgestreckten Bauch, dem ringförmilgen Fuß und dem niedrigen und weiten Hals soll auch dieses Gefäß wie die Trinkschale und der Napf aus diesem Grab die Metallvorbilder imitieren (Abb. 5, Zei. 5; H: 19,6 cm. T: hart gebrannt. Manche Teile des Gefäßes sind wegen der Verbrennung dunkelgrau-schwarzfarbig. Ziegelrötlicher Ton mit viel Sand und Kalkpartikeln. Ohne Bemalung und Überzug. B: abgesehen davon etwa $1 / 4$ der Mündung ganz erhalten. An der Mündung und auf dem Bauch sieht man Deformationen wegen der Verbrennung. Die Oberflächen der Außen- und Innenseiten, soweit man sieht, sind weitgehend mit Kalk bedeckt. Gleich unter der Verbindungsstelle des Halses und der Schulter sieht man eine plastische Linie). Besonders die dünnen und scharfkantigen Linien im Übergangsbereich vom Hals zur Schulter sowie die breite und stark betonte Lippe verstärken diese Vermutung. Der im Schnitt ellipsenförmige Henkel sitzt unten an der breitesten Stelle und verbreitert sich leicht nach außen verbiegt sich nach oben hin und verbindet sich oben mit einer leichten Krümmung mit der Lippe.

Das beste Vergleichsbeispiel, soweit mir bekannt ist, stammt aus Athen (Tuluk, 2003, 23). 
Besonders das Gefäß mit der Nummer 71 ähnelt mit seinem breiten und rundförmigen Bauch, dem gedrungenen und breiten Hals und mit dem Henkel, der sich oben direkt mit der Mündung verbindet, unserer Oinochoe. Der Henkel des 71 sitzt allerdings unten nicht an der breitesten Stelle, sondern am unteren Bereich der Schulter. Außerdem ist der Hals etwas niedriger als in unserem Beispiel.

Diese Form ist nach Fundkontexten ab etwa 275 v. Chr. in Gebrauch, allerdings die Form des Typs von 71 wird erst ab Ende des 3. Jhs v. Chr. hergestellt und ersetzen den Typ mit der glatten Standfläche. Allerdings berichtet Rotroff, dass die Oinochoe 71 mit seinem sehr niedrigen Hals und der Mündung charakteristisch am Ende des 2. Jhs. oder Anfang des 1. Jhs. hergestellt worden seien (Bailey, 1975, Pl. 112, No 584, 585, 588, 589, 590, 592. Das nächste Beispiel 590 wird ins späte 2 . Jh. v. Chr. datiert, während die anderen ins 1 . Jh. v. Chr. datiert werden). Diese Datierung passt allerdings nicht zur Datierung unseres Grabes. Die gut datierbare Münze erlaubt uns zu sagen, dass unsere Oinochoe etwas früher hergestellt wurde.

Ein anderes Vergleichsbeispiel stammt aus Sardes, wenn es auch nicht so nahe verwandt mit unserem Gefäß ist wie bei dem attischen Beispiel. Diese lydische Oinochoe hat ebenfalls einen breiten Bauch und einen Henkel, der unten an der breitesten Stelle des Bauches sitzt und sich oben mit der Mündung verbindet. Diese Oinochoe wurde mit der Kontext 1 zusammengefunden und wird daher vor 213 v. Chr. Datiert (Rotroff, 2006, 22, Abb. 3, no 71).

Allerdings können die Vergleichsbeispiele aus Sardes und Attika nicht viel zum Herstellungszentrum unserer Oinochoe beitragen. Die anderen Oinochoen aus Sardes und Attika unterscheiden sich eindeutig von unserem Gefäß. Auch die Datierungen dieser Oinochoen stimmen nicht richtig mit der Datierung unseres Grabes überein. Vor allem soll die Oinochoe 71 aus Athen mindestens 50 Jahre jünger sein als unser Gefäß. Die Oinochoe aus dem GörükleGrab soll wie die anderen Funde mit großer Wahrscheinlichkeit in der ersten Hälfte des 2. Jhs. hergestellt worden sein. Das Herstellungszentrum soll wegen der bescheidenen Qualität in einer Werkstatt in diesem Gebiet liegen.

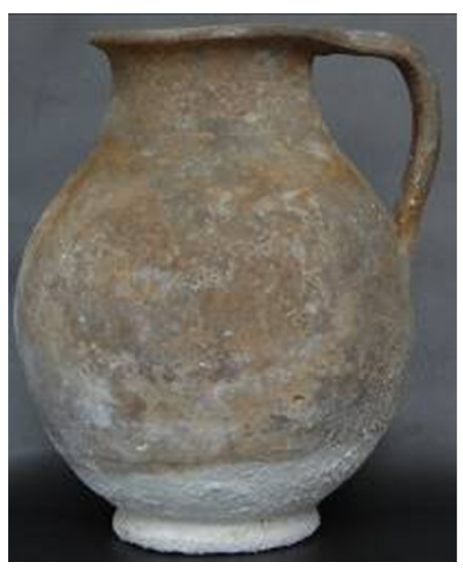

Abb. 5

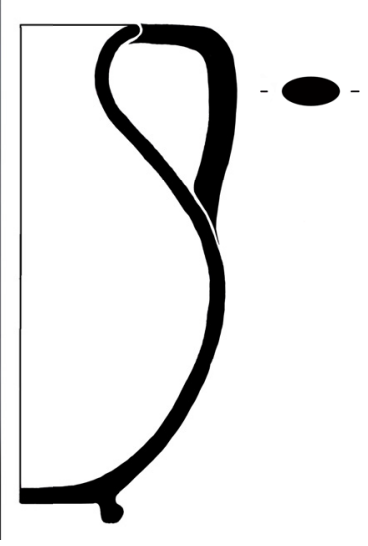

Zei. 5

6- Amphora erinnert mit ihrem ringförmigem $\mathrm{Fu} \beta$, dem breiten und rundförmigen Bauch, dem niedrigen und breiten Hals der bereits oben genannte Oinochoe (Abb. 6, Zei. 6; H: 19,6 cm. T: dunkelbräunlicher Ton, hart gebrannt. Ohne Bemalung und Überzug. B: ganz erhalten. Die Gefäßoberfläche ist weitgehend mit Kalk bedeckt. Die Außen- und Innenseiten der Mündung zeigen Rußspuren. Die Henkel bestehen aus je zwei im Schnitt rundförmigen Teilen und an der Verbindungsstelle an der Mündung haben sie an beiden Seiten Verbreiterungen. Im Gefäß befinden sich einige verbrannte Knochenreste). Auch die Form ihrer Henkel zeigen Ähnlichkeiten mit den Henkelformen dieser Oinochoe. Auch bei dieser Amphora sitzt der Henkel an der breitesten Stelle, verbreitert sich auch leicht nach Außen und verbindet sich 
direkt an der Mündung mit dem Gefäß. Außerdem unterscheidet sich Arbeits- und Tonqualität kaum von der Oinochoe. So liegt es nahe, dass beide Gefäße etwa gleichzeitig in derselben Werkstatt hergestellt wurden. Bei dieser Oinochoe wird der Übergang vom Hals zur Schulter anstatt einer konkaven Linie durch eine scharfkantige Verbindung des Halses und der Schulter betont. Ein weiterer Unterschied besteht darin, dass die Henkel sich aus je 2 im Schnitt rundförmigen, nebeneinander laufenden Teilen bestehen. Die breite Mündung hat in der Außenseite oben und unten je einen Vorsprung, deren Zwischenraum ein konkaves Profil hat. Somit unterscheidet sich zwar ihre Mündung von der Mündung der Oinochoe, jedoch sind beide schwer und stark betont und somit haben sie auch bei diesen Teilen Gemeinsamkeiten.

Soweit mir bekannt ist, kommt das beste Vergleichsbeispiel aus Athen, dessen Funde weitgehend und ausführlich publiziert wurden (Rotroff 2006, 78). Deren breiter und rundförmiger Bauch und der niedrige, breite, ringförmige Fuß zeigen große Ähnlichkeiten mit unserem Gefäß. An der Außenseite hat sie ebenfalls plastische, später hinzugefügte Vorsprünge, die oben und unten nach Außen gebeugt sind und dessen Zwischenraum ein konkaves Profil hat. Anders ist dagegen, dass der Henkel bei dieser Amphora im Schnitt platt ist und sich nicht in die Mündung, sondern etwa mittig des Halses verbindet. Diese Form aus Attika wird vom Verfasser wegen ihren vertikalen Henkeln als Amphora genannt und weil sie sehr selten in diesem Gebiet gefunden wurde und wegen den Ton-und Formunterschiede als Import bezeichnet. Die Höhen dieser Formen variieren sich zwischen 17 und $24 \mathrm{~cm}$. Unser Gefäß ist $20 \mathrm{~cm}$ hoch und passt somit genau zu der durchschnittlichen Höhe dieser Form in Athen. Da sie fürs Trinken eindeutig zu groß ist, sollen sie verwendet worden sein Oliven o. ä. kurzfristig aufzubewahren. Nach Fundkontexte werden diese Amphoren in Athen von der Mitte des 2. Jhds. v. Chr. bis 86 v. Chr. Datiert (Rotroff 2006, Fig. 21, no 132). Unsere Amphora dürfte wie die sehr ähnliche Oinochoe und andere Funde aus diesem Grab in der ersten Hälfte des 2. Jhs. v. Chr. hergestellt worden sein.

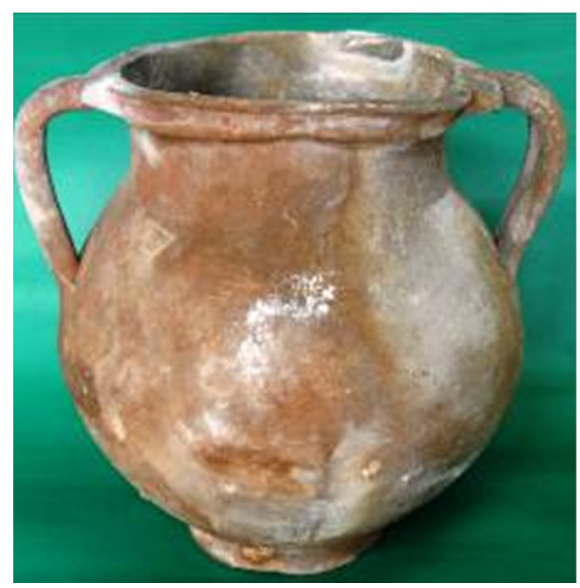

Abb. 6

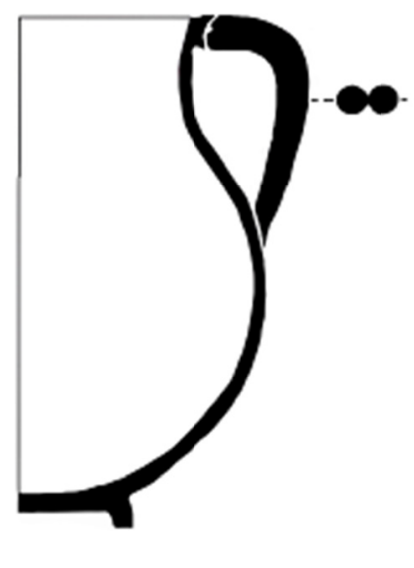

Zei. 6

\section{Danksagung}

Ich bedanke mich bei Frau Güler Ateş ganz herzlich für die Hinweise und Kritiken. 


\section{ABKÜRZUNGEN und QUELLEN}

Bailey, D. M. A. (1975). Catalogue of the Lamps in the British Museum I. London: British Museum Publications.

Bruneau, P. (1970). L'Ilot de la Maison des Comediens. La Vaiselle. Delos 27. Paris: De Boccard.

Bruneau, P. (1980). Aperçu sommaire sur la ceramique hellenistique. In Ceramiques Hellenistiques et Romaines, 9-15. Paris: Annales Litteraires de L'Universite de Besonçon.

Cook, R. M. (1966). Greek Painted Pottery. London: Methuen \& Co.

Doksanaltı, E. (2003). Knidos-Kap Krio Helenistik Sarnıç Buluntuları. In C. Abadie-Reynal (Hrgb.), Les Ceramiques en Anatolie aux Epoques Hellenistique ve Romaine, Kolloquium İstanbul- 1996 (27-35). Paris: De Boccard.

Dusinberre, E. R. M. (1999). Satrapal Sardis: Achaemenid Bowls in an Achaemenid Capital. American Journal of Archaeology, 103, 73-102.

Elaigne, S. (2002). L'Intoruduction des ceramiques fine hellenistiques du bassin oriental de la Mediterranee a Alexandrie. Importations et imitations locales. In Blonde, F-P. Ballet-J. F. Salles (Hrgb). Ceramiques hellenistiques et Romaines. Production et diffussion en Mediterranee orientale (Chypre, Egypte et cote syro-palestinienne), 159-173. Paris: De Boccard.

Gürler B. (2002). Tire-Ayaklıkırı'ndan bir Lagynos. Belleten, 65, 541-546.

Kögler, P. (2010). Feinkeramik aus Knidos vom mittleren Hellenismus bis in die mittlere Kaiserzeit. Wiesbaden: Warengruppe.

Mitsopoulos-Leon, V. (1991). Die Basilika am Staatsmarkt in Ephesos. Kleinfunde. 1. Teil: Keramik hellenistischer und römischer Zeit. Wien: Schindler.

Rotroff, S. I. (1997). Hellenistic Pottery. Athenian and Imported Wheelmade Table ware and related Material, The Athenian Agora 29. Princeton, N. J.: American School of Classical Studies at Athens.

Rotroff, S. I. (2006). Hellenistic Pottery. The Plain Ware. The Athenian Agora 33. Princeton, N. J.: American School of Classical Studies at Athens.

Rotroff, S. I., \& Oliver, A. Jr., (2003). The Hellenistic Pottery from Sardis. The Finds through 1994. Cambridge Mass.: Harvard University Press.

Schäfer, J. (1968). Hellenistische Keramik aus Pergamon, PF 2 . Berlin: Walter De Gruyter.

Schmidt, M. (1985). Die Vasenkunst des Hellenismus. Unpublished Dissertation, Berlin: FU.

Tuluk, G. G. (2003). İonia Bölgesi'ndeki Helenistik Döneme Ait Kandiller. In C. Abadie-Reynal (Hrgb.), Les Ceramiques en Anatolie aux Epoques Hellenistique ve Romaine, Kolloquium İstanbul- 1996, 17 25. Paris: De Boccard.

Vessberg, O., \& Westholm, A. (1956). The Hellenistic and Roman Periods in Cyprus. SCE IV, 3. Lund: Hakan Ohlssons Boktryckeri. 